\title{
Updated list of ant species (Hymenoptera, Formicidae) recorded in Santa Catarina State, southern Brazil, with a discussion of research advances and priorities
}

\author{
Mônica A. Ulysséa ${ }^{1}$, Carlos E. Cereto ${ }^{2}$, Félix B. Rosumek ${ }^{3}$, Rogério R. Silva ${ }^{4}$ \& Benedito C. Lopes ${ }^{3}$
}

${ }^{1}$ Laboratório de Entomologia da Universidade Estadual de Feira de Santana. Av. Transnordestina, s/n ${ }^{\circ}$. 44036-900 Feira de Santana-BA, Brasil. monicaulyssea@gmail.com

${ }^{2}$ Programa de Pós Graduação em Ecologia, Universidade Federal de Santa Catarina, 88040-970 Florianópolis-SC, Brasil. ducereto@gmail.com ${ }^{3}$ Departamento de Ecologia e Zoologia, Centro de Ciências Biológicas, Universidade Federal de Santa Catarina, 88040-970 Florianópolis-SC, Brasil. rosumek@ccb.ufsc.br; bclopes@ccb.ufsc.br

${ }^{4}$ Museu de Zoologia, Universidade de São Paulo, Seção de Entomologia. Av. Nazaré, 481, Caixa Postal 4249, 04263-000 São Paulo-SP, Brasil. rogeriorosas@gmail.com

\begin{abstract}
Updated list of ant species (Hymenoptera, Formicidae) recorded in Santa Catarina State, southern Brazil, with a discussion of research advances and priorities. A first working list of ant species registered in Santa Catarina State, southern Brazil was published recently. Since then, many studies with ants have been conducted in the state. With data compiled from published studies and collections in various regions of the state, we present here an updated list of 366 species (and 17 subspecies) in 70 ant genera in Santa Catarina, along with their geographical distribution in the seven state mesoregions. Two hundred and seven species are recorded in the Oeste mesoregion, followed by Vale do Itajaí (175), Grande Florianópolis (150), Norte (60), Sul (41), Meio Oeste (23) and Planalto Serrano (12). The increase in the number of records since 1999 results from the use of recently adopted sampling methods and techniques in regions and ecosystems poorly known before, and from the availability of new tools for the identification of ants. Our study highlights the Meio Oeste, Planalto Serrano, Sul and Norte mesoregions, as well as the deciduous forest, mangrove, grassland and coastal sand dune ecosystems as priority study areas in order to attain a more complete knowledge of the ant fauna in Santa Catarina State.
\end{abstract}

KEYWORDS. Formicidae; Santa Catarina State; species list.

RESUMO. Lista atualizada das espécies de formigas (Hymenoptera, Formicidae) registradas no Estado de Santa Catarina, sul do Brasil, com discussão sobre avanços e prioridades na pesquisa. Uma lista de espécies de formigas registradas em Santa Catarina foi publicada recentemente. Desde então, diversos estudos com formigas foram realizados em várias regiões do Estado. Neste trabalho, compilamos os dados de trabalhos publicados e de coletas avulsas, e apresentamos uma lista atualizada das espécies de formigas, com menção à distribuição geográfica nas sete mesorregiões de Santa Catarina. São registradas atualmente 366 espécies (e 17 subespécies) de formigas em 70 gêneros no território do Estado de Santa Catarina, sendo que a região Oeste apresenta o maior número de registros (207 espécies), seguida do Vale do Itajaí (175), Grande Florianópolis (150), Norte (60), Sul (41), Meio Oeste (23) e Planalto Serrano (12). O aumento no número de registros em relação à lista de 1999 deve-se principalmente à incorporação recente de métodos de coleta diferenciados e à realização de coletas em regiões e ecossistemas outrora subamostrados, além da disponibilidade de novas ferramentas de identificação. Nosso estudo aponta as mesorregiões Meio Oeste, Planalto Serrano, Sul e Norte, assim como os ecossistemas Florestas Estacionais Deciduais, Mangues, Campos e Restingas, como prioritárias para o aperfeiçoamento do banco de dados da mirmecofauna catarinense.

PALAVRAS-CHAVE. Formicidae; Santa Catarina; lista de espécies.

Although there are publications in Brazil with regional taxonomic lists for many animal groups, Santa Catarina is the only state with a list of ant species that occur within its borders. The ant fauna of the state of Santa Catarina has been studied since the beginning of the last century, mainly from sampling conducted by the naturalist, Fritz Plaumann, who lived in the district of Nova Teutônia, municipality of Seara, Oeste region of the state from 1924 until his death in 1994. During this period, Plaumann created an important collection of insects, including ants, making western Santa Catarina one of the most well known places with regard to Neotropical insects. Silva (1999) reviewed the literature at the time and the collection of the Museu de Zoologia, Universidade de São Paulo (MZSP) and listed a total of 342 species and subspecies of ants in the entire state of Santa Catarina, becoming an important reference for subsequent myrmecological studies.

Surveys of species and the creation of taxonomic lists are of fundamental importance for the development of scientific research and serve as a foundation for conservation projects. Species lists are critical to understanding biodiversity and species' distribution patterns, determining the main factors that threaten this biodiversity and establishing priorities for action (Marques et al. 2002; Lewinsohn et al. 2005). 
Lists of species occurrence are a key basis for management and conservation projects. Determining the area of distribution of a given species is one of the criteria used by the International Union for Conservation of Nature and Natural Resources for determining the level of threat to that species (IUCN 2001). These criteria have been used to create lists of endangered faunal species, especially vertebrates, in states in Brazil (Marques et al. 2002; Mikich \& Bérnils 2004). The inclusion of invertebrates in lists of threatened species is comparatively small due to lack of information on species distribution (see discussion in Brandão et al. 2008). In the list of threatened animal species in Rio Grande do Sul, for example, only 18 species of insects (of a total of 261 species) are listed, mostly bees and beetles, with no record of ants (Marques et al. 2002). In the list for Paraná, only bees and butterflies are included, accounting for 33 of a total of 163 threatened species (Mikich \& Bérnils 2004). On the national list, four ant species are considered endangered species among a large majority of other groups (MMA 2003, but see Brandão et al. 2008 for the case of Simopelta minima Brandão, 1989).

Over the past 11 years, several studies have been conducted involving ants in different regions of the state of Santa Catarina, led by groups of researchers from different universities and national research institutes. In this paper, we update the list of ant species known to exist in Santa Catarina, including new information from all recent studies involving ants, and review the current state of knowledge of the ant fauna in the state. We believe that our work may stimulate studies on the diversity, taxonomy and biogeography of the Neotropical ant fauna, in addition to promoting better-informed conservation policies in Santa Catarina State.

\section{MATERIAL AND METHODS}

This list of ant species for the state of Santa Catarina was made by adding data to the original list created by Silva (1999). These data were obtained from local studies involving ant fauna surveys conducted in several regions of the state (Lopes \& Fowler 2000; Silva \& Silvestre 2000, 2004; Lutinski \& Garcia 2005; Schmidt et al. 2005; Lutinski 2008; Rosumek et al. 2008; Iop et al. 2009; Cardoso \& Cristiano 2010; Cardoso et al. 2010; Silva \& Brandão 2010).

We also consulted the collection of the Laboratório de Biologia de Formigas, Universidade Federal de Santa Catarina for records not yet published, reviewing specimens of ants deposited by various collectors (listed in parentheses) from the following locations in the State: a) Oeste: Caçador (Hugo Borghezam Mozerle) and Piratuba (Carlos Eduardo Cereto); b) Planalto Serrano: Campo dos Padres/Urubici (Mônica Gomes) and Morro da Igreja/Urubici (Leonardo Wedekin); c) Vale do Itajaí: Camboriú and Blumenau (Mônica Antunes Ulysséa); d) Florianópolis: Anitápolis (Mônica Antunes Ulysséa), Antônio Carlos and Praia do Pântano do Sul, Florianópolis (Benedito Cortês Lopes); Lagoa do Peri/Florianópolis (Guilherme Willrich and Malva Isabel Medina Hernández),
Praia da Joaquina, Praia do Pântano do Sul, Ribeirão da Ilha e Trindade/Florianópolis (Mônica Antunes Ulysséa), Unidade de Conservação Desterro/Florianópolis (Carlos Eduardo Cereto) and Morro do Cambirela/Palhoça (Cássio Daltrini Neto).

The nomenclature adopted in the list follows Bolton et al. (2006), with the amendments proposed by Baroni-Urbani and Andrade (2007) for the tribe Dacetini, Klingenberg and Brandão (2009) for the Mycetophylax genus-group and La Polla et al. (2010) for the Prenolepis genus-group. The list incorporates taxonomic revisions of Cephalotes (De Andrade \& Baroni Urbani 1999), Carebara (Fernández 2004), Linepithema (Wild 2007), partially Trachymyrmex (MayhéNunes \& Brandão 2002, 2005, 2007) and Wasmannia (Longino \& Fernández 2007).

In the sum total of ant species for each mesoregion and for the state, we include subspecies names still recognized as valid, although there is a trend toward abandoning the use of subspecies in the taxonomy of ants (Bolton 2003). When only one subspecies is known for the state (as with Solenopsis decipiens abjecta Emery, 1906), this is counted as a species record.

\section{RESULTS}

Currently, there are 366 species and 17 subspecies of ants in 70 genera known for the state of Santa Catarina, with the mesoregion Oeste being the richest (with 207 species), followed by Vale do Itajaí (175 species), Grande Florianópolis (150 species), Norte (60 species), Sul (41 species), Meio Oeste (23 species) and Planalto Serrano (12 species) (Table I and Fig. 1).

Table I. Number of species and genera of Formicidae, by subfamily, recorded in Santa Catarina State, southern Brazil.

\begin{tabular}{lcc}
\hline \multicolumn{1}{c}{ Subfamily } & Species & Genera \\
\hline Amblyoponinae & 4 & 2 \\
Cerapachyinae & 7 & 4 \\
Dolichoderinae & 18 & 6 \\
Ecitoninae & 13 & 4 \\
Ectatomminae & 14 & 3 \\
Formicinae & 66 & 6 \\
Heteroponerinae & 8 & 2 \\
Myrmicinae & 189 & 32 \\
Ponerinae & 36 & 8 \\
Proceratiinae & 3 & 2 \\
Pseudomyrmecinae & 8 & 1 \\
\hline Total & 366 & 70 \\
\hline
\end{tabular}

Among the seven mesoregions of the state, specimens were recorded in the following locations:1) Oeste: Caçador, Chapecó, Concórdia, Seara (Nova Teutônia), Piratuba, Ponte Serrana, Quilombo, Seara, Xanxerê and Xaxim; 2) Meio Oeste: Ibicaré and Luzerna; 3) Planalto Serrano: Bom Retiro, 


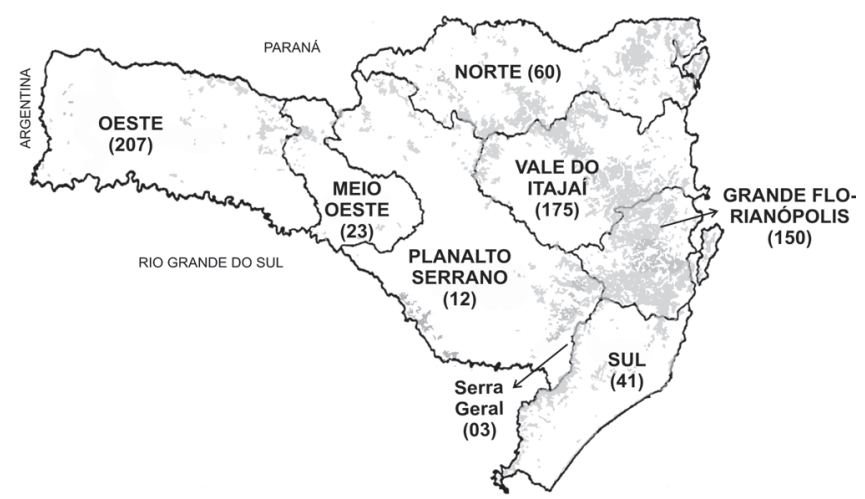

Fig. 1. Mesoregions of the state of Santa Catarina, southern Brazil, and the number of ant species recorded for each one. The gray areas indicate the remnants of ecosystems from the Atlantic rain forest domain in the state. Modified from Silva (1999) and Fundação SOS Mata Atlântica \& Instituto Nacional de Pesquisas Espaciais (2009).

Caçador, São Joaquim and Urubici; 4) Norte: Corupá, Jaraguá do Sul, Joinville and São Bento do Sul; 5) Vale do Itajaí: Balneário Camboriú, Blumenau, Brusque, Camboriú, Gaspar, Ibirama, Porto Belo (Ilha de João da Cunha), Ilhota, Itajaí, Itapema, Rio do Sul and Rodeio; 6) Grande Florianópolis: Anitápolis, Antônio Carlos, Florianópolis, Palhoça, Santo Amaro da Imperatriz, São Bonifácio and São José; 7) Sul: Araranguá (Morro dos Conventos), Criciúma and Urussanga.

The list of ant species for Santa Catarina and their distribution in the mesoregions of the state are shown below (Table II). The symbol, “?”, was used by Silva (1999) to indicate species recorded in the literature but not found by the author in the collection of the MZSP. Information for a few specimens does not indicate a specific location, only "region of the Serra Geral," which is not a mesoregion of the state; these entries are given separately.

\section{DISCUSSION}

In the eleven years between the list created by Silva (1999) and the updated list presented here, there has been an $11 \%$ increase in the number of species known for Santa Catarina. There was a higher increase in the number of species records in the regions of the state. Counted over all the regions, this number rose from 399 to 668 (not including the "SG" records), representing an increase of $66 \%$. Previously, most species were known from only one region of the state (mean of 1.2 record by species), but as expected, recent studies have contributed new data on species distribution (however, the mean remains low, with 1.8 record by species). Below, we discuss the factors that generated the increase in the number of records.

1. Collections in poorly sampled regions. Previously, the Oeste and Vale do Itajaí mesoregions contributed 45\% and $33 \%$, respectively, of the total of 399 records, with other mesoregions accounting for only $22 \%$ of this total. Currently, these two mesoregions still contribute the majority of the total of 668 records, but the proportion contributed has decreased to $31 \%$ and $26 \%$, respectively, indicating a more uniform distribution of the collections at Santa Catarina. The Oeste is still the best-sampled region of the state, notably through the work of the naturalist Fritz Plaumann (Silva 1999), and has also received recent contributions from Silva \& Silvestre (2000, 2004), Lutinski \& Garcia (2005), Lutinski (2008) and Iop et al. (2009).

The region of Grande Florianópolis has tripled its number of records, now accounting for $23 \%$ of the 668 records, mainly as a result of research carried out by the group of the Laboratório de Biologia de Formigas, Universidade Federal de Santa Catarina. The number of records from the Norte mesoregion has increased fivefold but does not yet represent the diversity expected for a region with a significant distribution of remnants of the lowland atlantic rain forest and Araucaria forest.

The expansion of knowledge about ants in the state can also be attributed to the number of sites studied. From 23 cities listed in Silva (1999), there are now specimens known from 42 cities. However, the mesoregions of Meio Oeste, Sul and Planalto Serrano remain clearly underrepresented. The recent papers of Cardoso \& Cristiano (2010) and Cardoso et al. (2010) present a series of new species records for $\operatorname{Sul}(38$, of which 9 are new for the state). For such underdocumented regions, rapid ecological assessments contribute a large number of new records, including common species that had not been recorded yet, as can be seen in the qualitative data gathered in Planalto Serrano. For the Meio Oeste mesoregion we have not found new data in our compilation, as there are no recent publications with the ant fauna in this region.

2. Collections in poorly-sampled ecosystems. Santa Catarina State contains a diversity of ecosystems and phytophysiognomies, some of which had not been adequately sampled. Recent collections in restingas (coastal ecosystems with sandy soils) and small islands have contributed to the increase in the number of records in Grande Florianópolis (Cereto, unpublished data), as well as in Sul (Cardoso \& Cristiano 2010; Cardoso et al. 2010).

In some ecosystems, such as the mangroves, grasslands and deciduous forests, the ant fauna is still poorly known. For example, a qualitative sampling of seven days in a deciduous forest area in Oeste (known locally as the Uruguay River forest), revealed 37 species and two new records (collected in Piratuba by C. E. Cereto in 2009). We consider this mesoregion relatively well-sampled, but the records were mostly from Araucaria forest.

3. New sampling techniques. In recent years, some studies have employed sampling techniques that were not used before to study the ant fauna of Santa Catarina, resulting in records of previously unknown species. We highlight the use of the Winkler extractor for sampling the fauna of litter, a new technique regarded as highly efficient for sampling cryptic ants (Delabie et al. 2000). A similar technique was used by Fritz Plaumann in his research on Oeste (Silva 1999), but for other regions, the use of the extractor is a novelty.

An example of the results produced by the use of complementary sampling techniques is the work of Rosumek et al. (2008) in the Atlantic rain forest, where were employed, for 
Table II. Ants recorded in Santa Catarina State, southern Brazil, listed alphabetically and by subfamily, tribe, genus, subgenus and species. Additions to the list by Silva (1999) are shown in bold. Mesoregions: $\mathrm{OE}=$ Oeste, $\mathrm{GF}=$ Grande Florianópolis, $\mathrm{MO}=$ Meio Oeste, $\mathrm{N}=$ Norte, $\mathrm{PS}=$ Planalto $\mathrm{Serrano}, \mathrm{S}=\mathrm{Sul}, \mathrm{VI}=\mathrm{Vale}$ do Itajaí, $\mathrm{SG}=\mathrm{Serra}$ Geral (location not specified), ? = specimens not deposited in MZSP, LD = unknown locality.

\begin{tabular}{|c|c|c|c|c|}
\hline Subfamily Tribe Genus (Subgenus) species & \multicolumn{4}{|c|}{ Mesoregions } \\
\hline \multicolumn{5}{|l|}{ Amblyoponinae } \\
\hline \multicolumn{5}{|l|}{ Amblyoponini } \\
\hline Amblyopone armigera Mayr, 1887 & MO & $\mathrm{OE}$ & GF & $\mathbf{N}$ \\
\hline A. degenerata Borgmeier, 1957 & $\mathrm{OE}$ & & & \\
\hline A. elongata (Santschi, 1912) & OE & GF & $\mathbf{N}$ & VI \\
\hline Prionopelta punctulata Mayr, 1866 & GF & $\mathbf{N}$ & & \\
\hline
\end{tabular}

\section{Cerapachyinae}

Acanthostichini

Acanthostichus flexuosus MacKay, 1996

A. quadratus Emery, 1895

$\mathrm{OE}$

A. serratulus (F. Smith, 1858)

$\mathrm{OE}$

Cerapachyini

Cerapachys splendens Borgmeier, 1957

C. toltecus Forel, 1909

Sphinctomyrmex stali Mayr, 1866

Cylindromyrmecini

Cylindromyrmex brasiliensis Emery, 1901

\begin{tabular}{|c|c|c|c|c|}
\hline \multicolumn{5}{|l|}{ Dolichoderinae } \\
\hline \multicolumn{5}{|l|}{ Dolichoderini } \\
\hline Anillidris bruchi Santschi, 1936 & $\mathrm{OE}$ & & & \\
\hline Azteca instabilis (F. Smith, 1862) & $?$ & & & \\
\hline A. lanuginosa Emery, 1893 & $?$ & & & \\
\hline A. mayrii Emery, 1893 & $?$ & & & \\
\hline A. muelleri Emery, 1893 & VI & & & \\
\hline Dolichoderus attelaboides (Fabricius, 1775) & VI & GF & & \\
\hline D. lobicornis (Kempf, 1959) & VI & & & \\
\hline Dorymyrmex brunneus (Forel, 1908) & $\mathrm{OE}$ & $\mathrm{S}$ & VI & GF \\
\hline D. pyramicus (Roger, 1863) & OE & $\mathbf{S}$ & & \\
\hline Linepithema angulatum (Emery, 1894) & VI & & & \\
\hline L. gallardoi (Brèthes, 1914) & $?$ & & & \\
\hline L. humile (Mayr, 1868) & GF & OE & PS & $\mathbf{S}$ \\
\hline L. iniquum (Mayr, 1870) & $\mathrm{OE}$ & VI & GF & $\mathbf{S}$ \\
\hline L. leucomelas (Emery, 1894) & $\mathrm{OE}$ & GF & & \\
\hline L. micans (Forel, 1908) & GF & PS & & \\
\hline L. neotropicum Wild, 2007 & $\mathbf{S}$ & & & \\
\hline Tapinoma atriceps Emery, 1888 & $\mathrm{OE}$ & VI & & \\
\hline T. melanocephalum (Fabricius, 1793) & GF & OE & & \\
\hline
\end{tabular}

\section{Ecitoninae}

Ecitonini

Eciton burchellii (Westwood, 1842)

E. burchellii foreli (Santschi, 1925)

E. quadriglume (Haliday, 1836)

Labidus coecus (Latreille, 1802)

L. praedator (F. Smith, 1858)

Neivamyrmex dorbignii (Shuckard, 1840)

N. halidaii (Shuckard, 1840)

N. hetschkoi (Mayr, 1886)

N. legionis (F. Smith, 1855)

N. minensis (Borgmeier, 1928)

N. punctaticeps (Emery, 1894)

N. spatulatus (Borgmeier, 1939)

MO OE $\quad$ N $\quad$ GF $\quad$ VI

OE

OE $\mathbf{N}$ GF

\begin{tabular}{lll}
\multicolumn{1}{c}{ Subfamily Tribe Genus (Subgenus) species } & & Mesoregions \\
\hline N. tenuis Borgmeier, 1953 & OE & \\
Nomamyrmex hartigii (Westwood, 1842) & N & OE \\
\hline
\end{tabular}

Ectatomminae

Ectatommini

Ectatomma edentatum Roger, 1863

GF OE VI

Gnamptogenys annulata (Mayr, 1887)

G. continua (Mayr, 1887)

G. lucaris Kempf, 1968

G. minuta (Emery, 1896)

G. moelleri (Forel, 1912)

G. rastrata (Mayr, 1866)

G. reichenspergeri (Santschi, 1929)

G. striatula Mayr, 1884

G. striolata (Borgmeier, 1957)

G. triangularis (Mayr, 1887)

VI

GF $\quad$ VI

MO VI

$\mathrm{OE}$

GF MO VI

OE $\mathrm{MO} \quad \mathbf{N}$ VI $\quad \mathbf{G F}$

OE VI GF

GF VI OE $\mathbf{S} \quad \mathbf{N}$

OE

OE

Typhlomyrmecini

Typhlomyrmex major (Santschi, 1923)

VI GF $\mathbf{N}$

T. pusillus Emery, 1894

OE VI N

T. rogenhoferi Mayr, 1862

Formicinae

Camponotini

Camponotus (Hypercolobopsis) divergens Mayr, 1887

C. (H.) paradoxus (Mayr, 1866)

LD

OE VI

C. (Myrmaphaenus) blandus (F. Smith, 1858) GF S

C. (M.) cameranoi (Emery, 1894) GF

C. (M.) fastigatus Roger, $1863 \quad$ GF VI

C. (M.) genatus Santschi, 1922 PS

C. (M.) iheringi Forel, $1908 \quad$ GF

C. (M.) integellus Forel, 1899

C. (M.) novogranadensis Mayr, $1870 \quad$ GF VI

C. (Myrmepomis) propinquus Mayr, $1887 \quad$ VI

C. (M.) scissus Mayr, 1887

C. (M.) sericeiventris (Guérin-Méneville, 1838) GF VI OE

C. (Myrmobrachys) arboreus (F. Smith, 1858) GF VI

C. (M.) brasiliensis Mayr, 1862

C. (M.) canescens Mayr, 1870 ?

C. (M.) caracalla Forel, 1912

C. (M.) crassus Mayr, $1862 \quad$ OE S

C. (M.) mus Roger, $1863 \quad$ OE

C. (M.) trapezoideus Mayr, $1870 \quad$ VI $\quad$ GF $\quad$ S

C. (Myrmocladoecus) bidens Mayr, $1870 \quad$ VI

C. (M.) hedwigae Forel, $1912 \quad$ OE VI

C. (M.) tripartitus Mayr, $1887 \quad$ VI

C. (Myrmosphincta) sexguttatus (Fabricius, 1793) VI

C. (Myrmothrix) atriceps (F. Smith, 1858) GF VI $\quad$ S

C. (M.) cingulatus Mayr, $1862 \quad$ VI

C. (M.) rufipes (Fabricius, 1775) GF OE PS S

C. (M.) sericatus Mayr, $1887 \quad$ LD

C. (Pseudocolobopsis) alboannulatus Mayr, $1887 \quad$ OE VI GF

C. (P.) macrocephalus (Erichson, 1842) OE VI

C. (P.) moelleri Forel, $1912 \quad$ VI

Continue next column 
Table II. Continued.

\begin{tabular}{|c|c|c|c|c|c|c|c|c|c|}
\hline Subfamily Tribe Genus (Subgenus) species & & & esoregions & Subfamily Tribe Genus (Subgenus) species & & & esoregi & ions & \\
\hline C. (Tanaemyrmex) balzani Emery, 1894 & VI & & & H. flava Kempf, 1962 & GF & $\mathbf{O E}$ & VI & & \\
\hline C. (T.) bonariensis Mayr, 1868 & GF & & & H. inermis (Emery, 1894) & $\mathrm{OE}$ & VI & GF & & \\
\hline C. (T.) bonariensis curtulus Santschi, 1936 & VI & & & H. mayri Kempf, 1962 & $\mathrm{OE}$ & VI & GF & $\mathbf{N}$ & \\
\hline C. (T.) bonariensis garbei Santschi, 1922 & $\mathrm{OE}$ & & & H. microps Borgmeier, 1957 & $\mathrm{OE}$ & & & & \\
\hline C. (T.) bonariensis parvulus (Emery, 1894) & $\mathrm{N}$ & $\mathrm{OE}$ & & Myrmicinae & & & & & \\
\hline C. (T.) diversipalpus Santschi, 1922 & OE & & & Adelomyrmecini & & & & & \\
\hline C. (T.) fuscocinctus (Emery, 1888) & $\mathrm{OE}$ & & & Cryptomyrmex boltoni (Fernández, 2003) & VI & & & & \\
\hline C. (T.) lespesii (Forel, 1886) & GF & & & Attini & & & & & \\
\hline C. (T.) melanoticus (Emery, 1894) & GF & $\mathrm{OE}$ & VI $\mathbf{S}$ & Acromyrmex (Acromyrmex) ambiguus (Emery, 1888) & $\mathbf{S}$ & & & & \\
\hline C. (T.) melanoticus catharinae Santschi, 1939 & VI & & & A. (A.) aspersus (F. Smith, 1858) & $\mathrm{OE}$ & VI & GF & $\mathbf{N}$ & \\
\hline C. (T.) melanoticus nigrescens Santschi, 1939 & VI & & & A. (A.) coronatus (Fabricius, 1804) & GF & VI & OE & & \\
\hline C. (T.) pallescens (Mayr, 1887) & $\mathrm{OE}$ & & & A. (A.) crassispinus (Forel, 1909) & GF & $\mathrm{OE}$ & PS & & \\
\hline C. (T.) punctulatus Mayr, 1868 & $\mathbf{S}$ & & & A. (A.) disciger (Mayr, 1887) & GF & VI & $\mathbf{O E}$ & PS & \\
\hline C. (T.) simillimum atratior Santschi, 1922 & VI & & & A. (A.) hispidus Santschi, 1925 & $\mathrm{OE}$ & MO & & & \\
\hline C. (T.) simillimus (F. Smith, 1862) & $\mathrm{OE}$ & VI & & A. (A.) hystrix (Latreille, 1802) & GF & & & & \\
\hline C. (T.) substitutus (Emery, 1894) & GF & & & A. (A.) laticeps (Emery, 1905) & $\mathrm{OE}$ & VI & & & \\
\hline C. (T.) xanthogaster Santschi, 1925 & $\mathrm{OE}$ & & & A. (A.) lundii (Guérin-Méneville, 1838) & PS & & & & \\
\hline C. (T.) zenon (Forel, 1912) & $\mathrm{OE}$ & VI & & A. (A.) niger (F. Smith, 1858) & GF & VI & OE & & \\
\hline Lasiini & & & & A. (A.) nigrosetosus (Forel, 1908) & GF & & & & \\
\hline Acropyga (Rhizomyrma) decedens (Mayr, 1887) & GF & & & A. (A.) rugosus (F. Smith, 1858) & GF & & & & \\
\hline A. (R.) goeldii Forel, 1893 & GF & & & A. (A.) subterraneus (Forel, 1893) & GF & VI & OE & & \\
\hline Myrmelachistini & & & & A. (A.) subterraneus brunneus (Forel, 1912) & $\mathrm{OE}$ & VI & & & \\
\hline Myrmelachista bambusarum Forel, 1903 & $\mathrm{OE}$ & & & A. (Moellerius) balzani (Emery, 1890) & GF & $\mathbf{S}$ & & & \\
\hline M. catharinae Mayr, 1887 & $\mathrm{OE}$ & GF & & A. (M.) fracticornis (Forel, 1909) & GF & & & & \\
\hline M. catharinae intermedia Santschi, 1929 & $\mathrm{OE}$ & VI & & A. (M.) heyeri (Forel, 1899) & $?$ & & & & \\
\hline M. catharinae maior Santschi, 1936 & VI & & & A. (M.) landolti (Forel, 1885) & $?$ & & & & \\
\hline M. gagatina Emery, 1894 & $?$ & & & A. (M.) striatus (Roger, 1863) & GF & $\mathbf{S}$ & & & \\
\hline M. gallicola Mayr, 1887 & $\mathbf{S}$ & & & Apterostigma mayri Forel, 1893 & $?$ & & & & \\
\hline M. kloetersi Forel, 1903 & $\mathrm{OE}$ & & & A. moelleri Forel, 1892 & $\mathrm{OE}$ & VI & & & \\
\hline M. nodigera Mayr, 1887 & $\mathrm{OE}$ & GF & & A. pilosum Mayr, 1865 & $\mathrm{OE}$ & & & & \\
\hline M. reticulata Borgmeier, 1928 & $\mathrm{OE}$ & & & A. wasmannii Forel, 1892 & $\mathrm{OE}$ & VI & & & \\
\hline M. ruszkii Forel, 1903 & $\mathrm{OE}$ & & & Atta (Neoatta) sexdens (Linnaeus, 1758) & $\mathrm{OE}$ & & & & \\
\hline Plagiolepidini & & & & Cyphomyrmex auritus Mayr, 1887 & VI & GF & & & \\
\hline Brachymyrmex (Brachymyrmex) admotus Mayr, 1887 & VI & GF & & C. hamulatus (Weber, 1938) & $\mathrm{OE}$ & & & & \\
\hline B. (B.) aphidicola (Forel, 1909) & $\mathrm{OE}$ & VI & & C. minutus Mayr, 1862 & $\mathrm{OE}$ & & & & \\
\hline B. (B.) coactus Mayr, 1887 & $\mathrm{OE}$ & GF & $\mathbf{N}$ & C. occultus Kempf, 1964 & $\mathrm{OE}$ & & & & \\
\hline B. (B.) cordemoyi (Forel, 1895) & $\mathrm{OE}$ & VI & $\mathbf{S}$ & C. olitor Forel, 1893 & $\mathrm{OE}$ & VI & $\mathbf{N}$ & & \\
\hline B. (B.) luederwaldti attenuatus Santschi, 1929 & VI & & & C. peltatus Kempf, 1966 & $\mathrm{MO}$ & $\mathrm{OE}$ & GF & & \\
\hline B. (B.) modestus Santschi, 1923 & VI & & & C. plaumanni Kempf, 1962 & $\mathrm{OE}$ & VI & $\mathbf{N}$ & & \\
\hline B. (B.) myops Emery, 1906 & $\mathrm{~N}$ & & & C. rimosus (Spinola, 1851) & VI & MO & $\mathrm{OE}$ & $\mathbf{S}$ & GF \\
\hline B. (B.) pictus Mayr, 1887 & ? & & & C. strigatus Mayr, 1887 & GF & $\mathbf{N}$ & $\mathbf{S}$ & VI & \\
\hline B. (B.) santschii Menozzi, 1927 & $\mathrm{OE}$ & & & Mycetarotes senticosus Kempf, 1960 & $\mathrm{OE}$ & & & & \\
\hline B. (Bryscha) pilipes Mayr, 1887 & $\mathrm{OE}$ & & & Mycetophylax morschi (Emery, 1888) & GF & $\mathbf{S}$ & & & \\
\hline Nylanderia docilis (Forel, 1908) & GF & VI & & M. simplex (Emery, 1888) & GF & $\mathbf{S}$ & & & \\
\hline N. fulva (Mayr, 1862) & GF & OE & & Mycetosoritis aspera (Mayr, 1887) & $\mathrm{OE}$ & & & & \\
\hline N. fulva fumatipennis (Forel, 1915) & ? & & & Mycocepurus goeldii (Forel, 1893) & $\mathrm{OE}$ & & & & \\
\hline Paratrechina longicornis (Latreille, 1802) & GF & OE & & Myrmicocrypta bruchi Santschi, 1936 & $\mathrm{OE}$ & & & & \\
\hline Heteroponerinae & & & & M. squamosa F. Smith, 1860 & GF & & & & \\
\hline Heteroponerini & & & & M. squamosa uncinata (Mayr, 1887) & $?$ & & & & \\
\hline Acanthoponera goeldii Forel, 1912 & $\mathrm{OE}$ & & & Trachymyrmex holmgreni W. M. Wheeler, 1925 & $\mathbf{S}$ & & & & \\
\hline A. mucronata (Roger, 1860) & GF & $\mathrm{OE}$ & VI & T. iheringi (Emery, 1888) & GF & OE & $\mathbf{S}$ & & \\
\hline Heteroponera dentinodis (Mayr, 1887) & GF & $\mathbf{N}$ & VI & Blepharidattini & & & & & \\
\hline H. dolo (Roger, 1860) & $\mathrm{OE}$ & VI & $\mathbf{N}$ & Wasmannia affinis Santschi, 1929 & GF & $\mathbf{S}$ & VI & & \\
\hline
\end{tabular}


Table II. Continued.

\begin{tabular}{|c|c|c|c|c|c|c|c|c|c|c|}
\hline Subfamily Tribe Genus (Subgenus) species & & & esoregi & sions & Subfamily Tribe Genus (Subgenus) species & & & soregi & ions & \\
\hline W. auropunctata (Roger, 1863) & $\mathrm{OE}$ & $\mathrm{S}$ & VI & GF & S. carinithorax Borgmeier, 1934 & GF & & & & \\
\hline W. lutzi Forel, 1908 & GF & & & & S. comis (Kempf, 1959) & SG & & & & \\
\hline W. sigmoidea (Mayr, 1884) & $?$ & & & & S. cordovensis Mayr, 1887 & $\mathrm{OE}$ & & & & \\
\hline W. sulcaticeps Emery, 1894 & $\mathbf{S}$ & & & & S. crassicornis Mayr, 1887 & $\mathrm{OE}$ & $\mathbf{N}$ & GF & $\mathbf{S}$ & VI \\
\hline Cephalotini & & & & & S. cultrigera (Mayr, 1887) & $\mathrm{OE}$ & & & & \\
\hline Cephalotes angustus (Mayr, 1862) & $\mathrm{OE}$ & VI & GF & & S. denticulata Mayr, 1887 & GF & $\mathrm{OE}$ & $\mathbf{S}$ & $\mathbf{N}$ & \\
\hline C. depressus (Klug, 1824) & $\mathrm{OE}$ & & & & S. eggersi Emery, 1890 & GF & & & & \\
\hline C. pallens (Klug, 1824) & $?$ & & & & S. elongata Roger, 1863 & GF & $\mathbf{N}$ & & & \\
\hline C. pallidicephalus (F. Smith, 1876) & GF & $\mathrm{OE}$ & VI & & S. fridericimuelleri Forel, 1886 & VI & & & & \\
\hline C. pinelii (Guérin-Méneville, 1844) & GF & MO & $\mathrm{OE}$ & VI & S. louisianae Roger, 1863 & $\mathrm{OE}$ & $\mathbf{N}$ & GF & $\mathbf{S}$ & VI \\
\hline C. pusillus (Klug, 1824) & $\mathrm{OE}$ & VI & & & S. lygatrix (Bolton, 2000) & VI & & & & \\
\hline Procryptocerus adlerzi (Mayr, 1887) & $\mathrm{OE}$ & PS & VI & GF & S. minuscula (Kempf, 1962) & $\mathrm{OE}$ & & & & \\
\hline P. clathratus Emery, 1896 & VI & & & & S. ogloblini Santschi, 1936 & GF & & & & \\
\hline P. convergens (Mayr, 1887) & GF & $\mathrm{OE}$ & VI & & S. perparva Brown, 1958 & GF & & & & \\
\hline P. goeldii Forel, 1899 & $\mathrm{OE}$ & VI & GF & & S. rugithorax (Kempf, 1959) & $\mathrm{OE}$ & $\mathbf{N}$ & GF & VI & \\
\hline P. lenkoi Kempf, 1969 & $\mathrm{OE}$ & & & & S. saliens Mayr, 1887 & $\mathrm{OE}$ & VI & GF & & \\
\hline P. lepidus (Forel, 1908) & $\mathrm{OE}$ & & & & S. schmalzi Emery, 1906 & GF & $\mathrm{N}$ & & & \\
\hline P. regularis (Emery, 1888) & $\mathrm{OE}$ & VI & & & S. silvestrii Emery, 1906 & $\mathrm{OE}$ & & & & \\
\hline P. sampaioi Forel, 1912 & VI & GF & & & S. smithii Forel, 1886 & VI & & & & \\
\hline P. schmalzi (Emery, 1894) & $\mathrm{N}$ & $\mathrm{OE}$ & & & S. splendens (Borgmeier, 1954) & $\mathrm{OE}$ & $\mathbf{N}$ & & & \\
\hline P. seabrai Kempf, 1964 & GF & & & & S. subedentata Mayr, 1887 & GF & $\mathbf{N}$ & & & \\
\hline Crematogastrini & & & & & S. tanymastax (Brown, 1964) & $\mathrm{OE}$ & & & & \\
\hline Crematogaster (Eucrema) acuta (Fabricius, 1804) & MO & VI & $\mathbf{O E}$ & & Formicoxenini & & & & & \\
\hline C. (E.) bingo Forel, 1908 & $\mathrm{OE}$ & & & & Nesomyrmex asper (Mayr, 1887) & VI & & & & \\
\hline C. (Neocrema) corticicola (Mayr, 1887) & $\mathrm{OE}$ & & & & N. costatus (Emery, 1896) & VI & & & & \\
\hline C. (N.) magnifica Santschi, 1925 & $\mathrm{OE}$ & & & & N. schwebeli (Forel, 1913) & $\mathrm{OE}$ & & & & \\
\hline C. (N.) montezumia F. Smith, 1858 & VI & & & & N. sculptiventris (Mayr, 1887) & LD & & & & \\
\hline C. (O.) crinosa Mayr, 1862 & $\mathrm{OE}$ & & & & N. vicinus (Mayr, 1887) & $\mathrm{OE}$ & & & & \\
\hline C. (O.) curvispinosa Mayr, 1862 & $\mathrm{OE}$ & VI & & & Myrmicini & & & & & \\
\hline C. (O.) limata (F. Smith, 1858) & VI & GF & $\mathbf{O E}$ & & Hylomyrma balzani (Emery, 1894) & $\mathrm{OE}$ & & & & \\
\hline C. (O.) lutzi Forel, 1905 & $\mathrm{OE}$ & VI & & & H. reitteri (Mayr, 1887) & GF & $\mathrm{OE}$ & VI & $\mathbf{N}$ & \\
\hline C. (O.) moelleri (Forel, 1912) & $\mathrm{OE}$ & VI & $\mathbf{S}$ & & Pogonomyrmex naegelii Forel, 1878 & GF & OE & PS & $\mathbf{S}$ & \\
\hline C. (O.) nigropilosa Mayr, 1870 & VI & GF & $\mathbf{O E}$ & & Pheidolini & & & & & \\
\hline C. (O.) parallela Santschi, 1925 & VI & & & & Pheidole (Elasmopheidole) aberrans Mayr, 1868 & $\mathrm{OE}$ & & & & \\
\hline C. (O.) quadriformis Roger, 1863 & $?$ & & & & P. (E.) weiseri (Santschi, 1923) & $?$ & & & & \\
\hline C. (O.) tenuicula (Forel, 1904) & LD & & & & P. (Pheidole) auropilosa Mayr, 1887 & $\mathrm{OE}$ & & & & \\
\hline Dacetini & & & & & P. (P.) bambusarum Forel, 1908 & $\mathrm{OE}$ & & & & \\
\hline Acanthognathus brevicornis M. R. Smith, 1944 & $\mathbf{N}$ & & & & P. (P.) blumenauensis Kempf, 1964 & VI & & & & \\
\hline A. ocellatus Mayr, 1887 & $\mathrm{OE}$ & VI & & & P. (P.) brevicona (Mayr, 1887) & $\mathrm{OE}$ & & & & \\
\hline A. rudis Brown \& Kempf, 1969 & $\mathrm{OE}$ & VI & GF & & P. (P.) dyctiota Kempf, 1972 & $\mathrm{OE}$ & & & & \\
\hline Basiceros acutipilis (Kempf, 1962) & $\mathrm{OE}$ & & & & P. (P.) fabricator (F. Smith, 1858) & VI & & & & \\
\hline B. bruchi (Santschi, 1922) & $\mathbf{N}$ & & & & P. (P.) flavens Roger, 1863 & $\mathrm{OE}$ & VI & LD & & \\
\hline B. convexiceps (Mayr, 1887) & $\mathbf{N}$ & OE & & & P. (P.) flavida Mayr, 1887 & VI & & & & \\
\hline B. disciger (Mayr, 1887) & $\mathrm{OE}$ & VI & GF & $\mathbf{N}$ & P. (P.) gertrudae Forel, 1886 & $?$ & & & & \\
\hline B. iheringi (Emery, 1888) & GF & & & & P. (P.) gibba Mayr, 1887 & VI & & & & \\
\hline B. petiolatum (Mayr, 1887) & GF & $\mathbf{N}$ & & & P. (P.) guilelmimuelleri Forel, 1886 & $\mathrm{OE}$ & VI & LD & & \\
\hline B. plaumanni (Brown \& Kempf, 1960) & $\mathrm{OE}$ & & & & P. (P.) heyri (Forel, 1899) & VI & & & & \\
\hline B. rugiferum (Mayr, 1887) & $\mathrm{OE}$ & VI & GF & $\mathbf{N}$ & P. (P.) laevifrons Mayr, 1887 & LD & & & & \\
\hline B. speciosum (Brown \& Kempf, 1960) & $\mathrm{OE}$ & & & & P. (P.) lignicola Mayr, 1887 & LD & & & & \\
\hline B. spectabile (Kempf, 1962) & $\mathrm{OE}$ & $\mathbf{N}$ & & & P. (P.) lucretii Santschi, 1923 & VI & & & & \\
\hline B. stenognathum (Brown \& Kempf, 1960) & GF & $\mathrm{OE}$ & VI & $\mathbf{N}$ & P. (P.) megacephala (Fabricius, 1793) & GF & & & & \\
\hline Phalacromyrmex fugax Kempf, 1960 & MO & GF & $\mathbf{N}$ & & P. (P.) nana Emery, 1894 & $\mathrm{OE}$ & VI & & & \\
\hline Strumigenys appretiata (Borgmeier, 1954) & $\mathrm{OE}$ & VI & GF & & P. (P.) peregrina W. M. Wheeler, 1916 & $\mathrm{OE}$ & & & & \\
\hline
\end{tabular}


Table II. Continued.

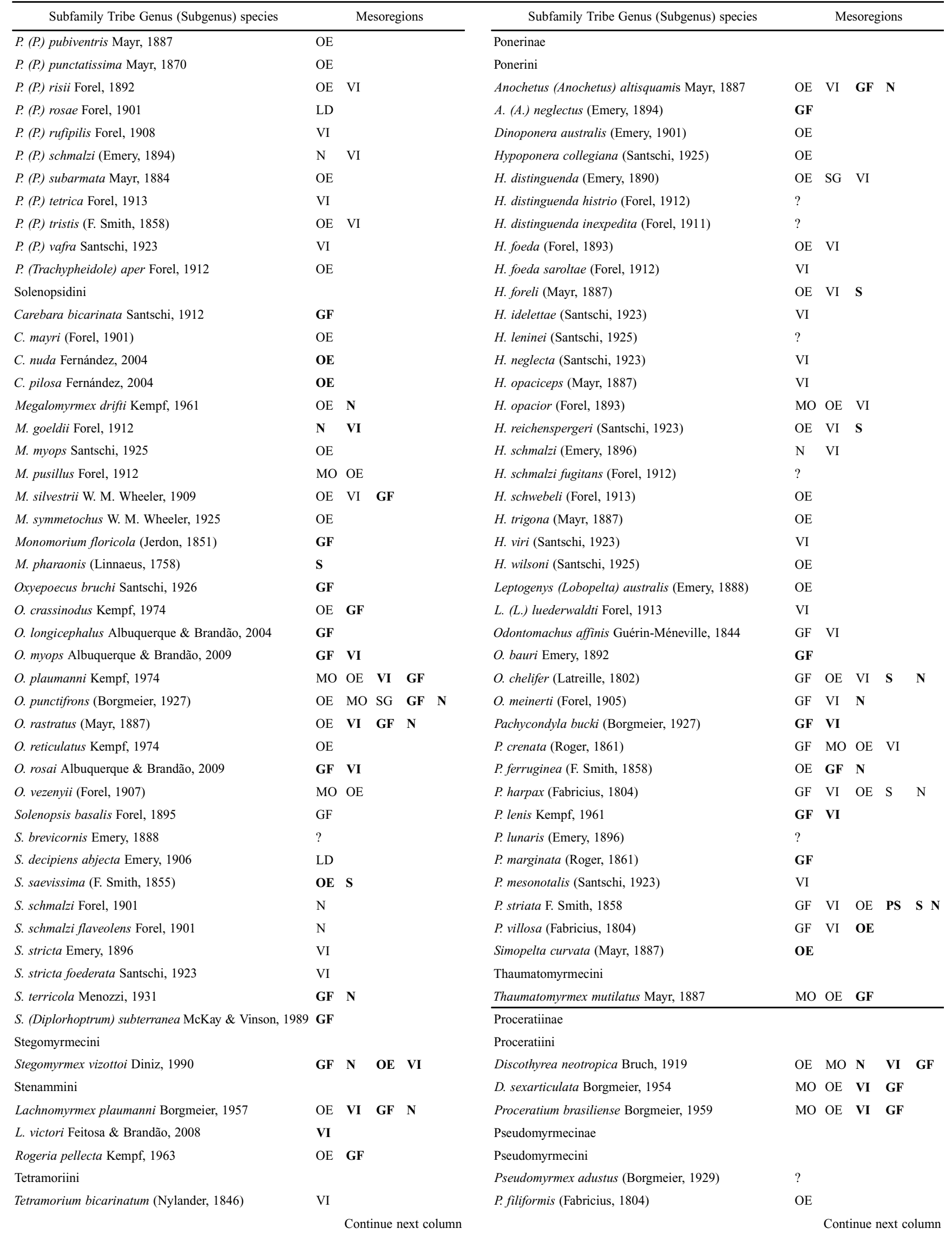


Table II. Continued.

\begin{tabular}{|c|c|c|c|c|c|}
\hline Subfamily Tribe Genus (Subgenus) species & & Mesoregions & Subfamily Tribe Genus (Subgenus) species & & Mesoregions \\
\hline P. flavidulus (F. Smith, 1858) & GF & OE VI & P. pisinnus Ward, 1989 & GF & \\
\hline P. gracilis (Fabricius, 1804) & GF & OE VI & P. schuppi (Forel, 1901) & $\mathrm{S}$ & VI \\
\hline \multirow[t]{2}{*}{ P. phyllophilus (F. Smith, 1858) } & GF & MO OE VI & P. termitarius (F. Smith, 1855) & PS & \\
\hline & & Continue next $\mathrm{c}$ & & & \\
\hline
\end{tabular}

the fist time in Grande Florianópolis, three techniques (Winkler extractor, pitfall traps and sampling in bromeliads), revealing 30 new records for the mesoregion (of which 11 were new for the state), including several small and cryptic ants.

The use of complementary techniques of collection, even in well-sampled regions, reveals undiscovered species that occupy specialized niches in communities, and is therefore highly recommended. The subterranean ant fauna, for example, has a potentially high richness (Longino \& Colwell 1997) and is almost unknown in the state, about which there has been only one recent paper (Silva \& Silvestre 2004). The subterranean ant fauna has great potential for revealing taxonomic novelties, and the few subterranean species recorded so far represent incidental cases of specimens visiting the surface.

4. Recent taxonomic reviews and new keys for species identification. The identification of ant genera in the Neotropical region is a relatively easy task thanks to the existence of guides and regular updates (e.g., Bolton 1994; Palácio \& Fernández 2003). There are new tools for the identification of ants from the Neotropical region, including image databases and electronic keys (e.g., Pheidole, Longino 2009).

In the last decade, several genera of wide distribution have been reviewed (see Materials and Methods). These works allow researchers to review specimens collected before and assign names to the morphospecies recorded in Santa Catarina. However, species identification is still a difficult task for some genera, such as Brachymyrmex and Camponotus (Formicinae), Crematogaster (see Longino 2003 for species of Costa Rica and problems with synonymies), Pheidole and Solenopsis (Myrmicinae), Hypoponera (Ponerinae) and Pseudomyrmex (Pseudomyrmecinae).

In such cases, the only way to identify species is through direct comparison with specimens deposited in established collections and the study of original descriptions, which becomes especially difficult when it comes to genera with great richness or with unknown species for a given region. As a result, all individuals remain identified as morphospecies, which are useful for a number of other studies, but add little to the taxonomic knowledge in the form of ant species lists. A clear example is the Solenopsis, with only 10 species/ subspecies recorded for Santa Catarina, but with a greater number of morphospecies found in recent studies (Silva \& Silvestre 2004; Rosumek et al. 2008). Certainly, the richness and distribution of species of these genera are underestimated in the present list, a problem common to all listings of ants.

Priority areas for inventory studies. Originally, the state of Santa Catarina was fully covered by vegetation communities of the Atlantic rain forest domain, including pioneer formations (mangroves and restingas), rain forests (lowland rain forest and upper montane rain forest), evergreen coniferous forests (Araucaria forest and faxinal forest), grasslands and deciduous forests (Uruguay River forest) (FATMA 2001).

In three years, from 2005 to 2008, Santa Catarina lost 25,953 hectares of Atlantic rain forest, a loss smaller only than that in the state of Minas Gerais, with 32,728 ha deforested. Today, the total area has shrunk to $23.4 \%$ of its original coverage in the state (Fundação SOS Mata Atlântica \& Instituto Nacional de Pesquisas Espaciais 2009). Among the seven mesoregions of the state, the remnants are concentrated in the Grande Florianópolis, Vale do Itajaí, Norte and near the mountain ridges of Serra Geral (which includes part of the Planalto Serrano and Sul) (Fig. 1). The Norte and the Serra Geral are recommended areas for sampling, due to the presence of these remnants. We consider the lowland rain forests of Grande Florianópolis well known, but it should be noted that the majority of the samples have been made in the Ilha de Santa Catarina. The continental portion has a high concentration of remnants, including primary ones, and still offer good possibilities of taxonomic discoveries. Also, the Meio Oeste and Planalto Serrano, even with few remnants, are so critically underdocumented that collections of ants in these areas are recommended.

In addition to this geographic approach, attention should be given to the poorly known ecosystems, including the deciduous forests, upper montane rain forests, faxinal forests, mangroves, grasslands and restingas. In relatively wellsampled locations (the Araucaria forests in Oeste and lowland rain forests in Vale do Itajaí and Grande Florianópolis) methods and techniques not previously employed should be prioritized.

\section{CONCLUSIONS}

Clearly, from the current revision of the ant species list in Santa Catarina, a major effort will be necessary to obtain more complete information for the state. We have seen a meaningful improvement in knowledge, in part due to the existence of this updated species list, with its regional refinement, which will allow future studies to more easily compare their findings with previous records. We expect this update to help solve the critical issues raised above, and to help stimulate the production of similar syntheses for other states, regions or ecosystems in Brazil and abroad. 


\section{ACKNOWLEDGEMENTS}

We thank Carlos Roberto F. Brandão for the use of the ant collection at the Museu de Zoologia, Universidade de São Paulo. We also thank Rodrigo M. Feitosa and Carlos Roberto F. Brandão for critical reading of previous versions of the manuscript.

\section{REFERENCES}

Baroni-Urbani, C. \& M. L. de Andrade. 2007. The ant tribe Dacetini: limits and constituent genera, with descriptions of new species (Hymenoptera, Formicidae). Annali del Museo Civico di Storia Naturale "Giacomo Doria" 95: 1-191.

Bolton, B. 1994. Identification guide to the ant genera of the world. Cambridge, Harvard University Press, 296 p.

Bolton, B. 2003. Synopsis and classification of Formicidae. Memoirs of the American Entomological Institute 71: 1-370.

Bolton, B.; G. Alpert; P. S. Ward \& P. Naskrecki. 2006. Bolton's Catalogue of Ants of the World 1758-2005. Cambridge, Harvard University Press, CD-ROM.

Brandão, C. R. F.; R. M. Feitosa; F. A. Schmidt \& R. R. E. Solar. 2008. Rediscovery of the putatively extinct ant species Simopelta minima (Brandão) (Hymenoptera, Formicidae), with a discussion on rarity and conservation status of ant species. Revista Brasileira de Entomologia 52: $480-483$.

Cardoso, D. C. \& M. P. Cristiano. 2010. Myrmecofauna of the southern Catarinense Restinga Sandy Coastal Plain: New records of species occurrence for the state of Santa Catarina and Brazil. Sociobiology 55: 229-239.

Cardoso, D. C.; T. G. Sobrinho \& J. H. Schoereder. 2010. Ant community composition and its relationship with phytophysiognomies in a Brazilian Restinga. Insectes Sociaux 57: 1-9.

De Andrade, M. L. D. \& C. Baroni-Urbani. 1999. Diversity and adaptation in the ant genus Cephalotes, past and present. Stuttgarter Beiträge zur Naturkunde B 271: 1-889.

Delabie, J. H. C; B. L. Fisher; J. D. Majer \& I. W. Wright. 2000. Sampling effort and choice of methods, p. 145-154. In: D. Agosti; J. D. Majer; L. E. Alonso \& T. R. Schultz (eds.). Ants: Standard methods for measuring and monitoring biodiversity. Washington Smithsonian Institution Press, $280 \mathrm{p}$.

FATMA/Fundação do Meio Ambiente. 2001. Mapa fitogeográfico com informações políticas do Estado de Santa Catarina. Available from: http://www.fatma.sc.gov.br/(accessed 4 March 2009).

Fernández, F. 2004. The American species of the myrmicine ant genus Carebara Westwood (Hymenoptera: Formicidae). Caldasia 26: 191-238.

Fundação SOS Mata Atlântica \& Instituto Nacional de Pesquisas Espaciais. 2009. Atlas dos Remanescentes Florestais da Mata Atlântica, Período 2005-2008. Relatório Parcial. Available from: http:// www.sosmatatlantica.org.br (accessed 4 March 2009).

Iop, S.; V. M.Caldart; J. A. Lutinski \& F. R. M. Garcia. 2009. Formigas urbanas da cidade de Xanxerê, Santa Catarina, Brasil. Biotemas 22: 55-64.

IUCN/International Union for Conservation of Nature and Natural Resources. 2001. IUCN Red List Categories and Criteria: Version 3.1. IUCN, Gland, Switzerland and Cambridge, IUCN Species Survival Commission, $\mathrm{ii}+30 \mathrm{p}$.

Klingenberg, C. \& C. R. Brandão. 2009. Revision of the fungus-growing ant genera Mycetophylax Emery and Paramycetophylax Kusnezov rev. stat., and description of Kalathomyrmex n. gen. (Formicidae: Myrmicinae: Attini). Zootaxa 2052: 1-31.

La Polla, J. S.; S. G. Brady \& S. O. Shattuck. 2010. Phylogeny and taxonomy of the Prenolepis genus-group of ants (Hymenoptera: Formicidae). Systematic Entomology 35: 118-131.

Lewinsohn, T. M.; A. V. L. Freitas \& P. I. Prado. 2005. Conservation of terrestrial invertebrates and their habitats in Brazil. Conservation Biology 19: 640-645.
Longino, J. T. \& R. K. Colwell. 1997. Biodiversity assessment using structured inventory: capturing the ant fauna of a tropical rain forest. Ecological Applications 7: 1263-1277.

Longino, J. T. \& F. Fernández. 2007. Taxonomic review of the genus Wasmannia, p. 271-289. In: Snelling, R. R.; B. L. Fisher \& P. S. Ward. (eds.). Advances in ant systematics (Hymenoptera: Formicidae): Homage to E. O. Wilson - 50 years of contributions. Gainesville, American Entomological Institute, 690 p.

Longino, J. T. 2003. The Crematogaster (Hymenoptera, Formicidae, Myrmicinae) of Costa Rica. Zootaxa 151: 1-150.

Longino, J. T. 2009. Additions to the taxonomy of New World Pheidole. Zootaxa 2181: 1-90.

Lopes, B. C. \& H. G. Fowler. 2000. Fungus-growing ants (Hymenoptera: Formicidae) on Santa Catarina Island, Brazil: patterns of occurrence. Revista de Biología Tropical 48: 643-646.

Lutinski, J. A. 2008. Diversidade de formigas na Floresta Nacional de Chapecó, Santa Catarina, Brasil. Ciência Rural 38: 1810-1816.

Lutinski, J. A. \& F. R. M. Garcia. 2005. Análise faunística de Formicidae (Hymenoptera: Apocrita) em ecossistema degradado no município de Chapecó, Santa Catarina. Biotemas 18: 73-86.

Marques, A. A. B.; C. S. Fontana; E. Velez; G. A. Bencke; M. Schneider \& R. E. Reis, (orgs.). 2002. Lista de Referência da Fauna Ameaçada de Extinção no Rio Grande do Sul - Decreto No 41.672, de 11 de Junho de 2002. Porto Alegre, FZB/MCT-PUCRS/PANGEA, 52 p.

Mayhé-Nunes, A. J. \& C. R. F. Brandão. 2002. Revisionary studies on the attine ant genus Trachymyrmex Forel. Part 1: Definition of the genus and the Opulentus group (Hymenoptera: Formicidae). Sociobiology 40: 667-698.

Mayhé-Nunes, A. J. \& C. R. F. Brandão. 2005. Revisionary studies on the attine ant genus Trachymyrmex Forel. Part 2: The Iheringi group (Hymenoptera: Formicidae). Sociobiology 45: 271-305.

Mayhé-Nunes, A. J. \& C. R. F. Brandão. 2007. Revisionary studies on the attine ant genus Trachymyrmex Forel. Part 3: The Jamaicensis group (Hymenoptera: Formicidae). Zootaxa 1444: 1-27.

Mikich, S.B. \& R. S. Bérnils. 2004. Livro Vermelho da Fauna Ameaçada no Estado do Paraná. Available from: http://www.pr.gov.br/iap (accessed 4 March 2009).

MMA/Ministério do Meio Ambiente. 2003. Lista Nacional das Espécies da Fauna Brasileira Ameaçadas de Extinção. Available from: www.mma.gov.br (accessed 4 March 2009).

Palácio, E. E. \& F. Fernández. 2003. Claves para las subfamilias y gêneros, p. 233-260. In: F. Fernández (ed.). Introducción a las hormigas de la región Neotropical. Bogotá, Instituto de Investigación de Recursos Biológicos Alexander Von Humboldt. xxvi+398 p.

Rosumek, F. B.; M. A. Ulysséa; B. C. Lopes; J. Steiner \& A. Zillikens. 2008. Formigas de solo e de bromélias em uma área de Mata Atlântica, Ilha de Santa Catarina, sul do Brasil: Levantamento de espécies e novos registros. Biotemas 21: 81-89.

Schmidt, K.; R. Corbetta \& A. J. A. de Camargo. 2005. Formigas (Hymenoptera: Formicidae) da Ilha João da Cunha, SC: composição e diversidade. Biotemas 18: 57-71.

Silva, R. R. 1999. Formigas (Hymenoptera: Formicidae) do oeste de Santa Catarina: Histórico de coletas e lista atualizada das espécies do Estado de Santa Catarina. Biotemas 12: 75-100.

Silva, R. R. \& C. R. F. Brandão. 2010. Morphological patterns and community organization in leaf-litter ant assemblages. Ecological Monographs 80: 107-124.

Silva, R. R. \& R. Silvestre. 2000. Diversidade de formigas em Seara, Oeste de Santa Catarina. Biotemas 13: 85-105.

Silva, R. R. \& R. Silvestre. 2004. Riqueza da fauna de formigas (Hymenoptera: Formicidae) que habita as camadas superficiais do solo em Seara, Santa Catarina. Papéis Avulsos de Zoologia 44: 1-11.

Wild, A. L. 2007. Taxonomic revision of the ant genus Linepithema (Hymenoptera: Formicidae). University of California Publications in Entomology 126: 1-151.

Received 29/12/2010; accepted 2/12/2011

Editor: Márcio Roberto Pie 